\title{
A SELF-REGULATING AND PATCH SUBDIVIDED POPULATION
}

\author{
LAMIA BELHADJI, ${ }^{*}$ Université de Rouen \\ DANIELA BERTACCHI, ${ }^{* *}$ Universitá di Milano-Bicocca \\ FABIO ZUCCA, ${ }^{* * *}$ Politecnico di Milano
}

\begin{abstract}
We consider an interacting particle system on a graph which, from a macroscopic point of view, looks like $\mathbb{Z}^{d}$ and, at a microscopic level, is a complete graph of degree $N$ (called a patch). There are two birth rates: an inter-patch birth rate $\lambda$ and an intra-patch birth rate $\phi$. Once a site is occupied, there is no breeding from outside the patch and the probability $c(i)$ of success of an intra-patch breeding decreases with the size $i$ of the population in the site. We prove the existence of a critical value $\lambda_{\mathrm{c}}(\phi, c, N)$ and a critical value $\phi_{\mathrm{c}}(\lambda, c, N)$. We consider a sequence of processes generated by the families of control functions $\left\{c_{n}\right\}_{n \in \mathbb{N}}$ and degrees $\left\{N_{n}\right\}_{n \in \mathbb{N}}$; we prove, under mild assumptions, the existence of a critical value $n_{\mathrm{c}}(\lambda, \phi, c)$. Roughly speaking, we show that, in the limit, these processes behave as the branching random walk on $\mathbb{Z}^{d}$ with inter-neighbor birth rate $\lambda$ and on-site birth rate $\phi$. Some examples of models that can be seen as particular cases are given.
\end{abstract}

Keywords: Contact process; restrained branching random walk; epidemic model; phase transition; critical parameters

2010 Mathematics Subject Classification: Primary 60K35

\section{Introduction}

Stochastic models for the demographic expansion (or contraction) of a biological population have attracted the attention of many researchers since Galton and Watson introduced the branching process as a very simple and nonspatial model to study the survival of surnames. After the appearance of interacting particle systems and of the paper by Harris [10] on the contact process, it has become increasingly clear that the spatial structure of the environment and the interaction between individuals bring a desirable complexity to the models one can define (see, e.g. [9] for a discussion on spatial models).

We want to introduce and study a model for a biological population where individuals breed and die living on a patchy habitat. Clearly, if we substitute the words infect and recover for breed and die, our model also serves as a picture for the spreading of an infectious disease.

The main idea is that the environment is subdivided into patches, the patches are centered at the vertices of the $d$-dimensional lattice $\mathbb{Z}^{d}$, each patch has $N$ sites and each site may host a colony of one or more particles (see Figure 1, where each square is a patch, $N=9$, and

\footnotetext{
Received 20 April 2009; revision received 26 April 2010.

* Postal address: Laboratoire de Mathématiques Raphaël Salem, UMR 6085, CNRS - Université de Rouen, Avenue de l’Université, BP. 12, 76801 Saint Etienne du Rouvray, France. Email address: lamia.belhadji@univ-rouen.fr

** Postal address: Universitá di Milano-Bicocca Dipartimento di Matematica e Applicazioni, Via Cozzi 53, 20125 Milano, Italy. Email address: daniela.bertacchi@unimib.it

*** Postal address: Dipartimento di Matematica, Politecnico di Milano, Piazza Leonardo da Vinci 32, 20133 Milano, Italy. Email address: fabio.zucca@polimi.it
} 


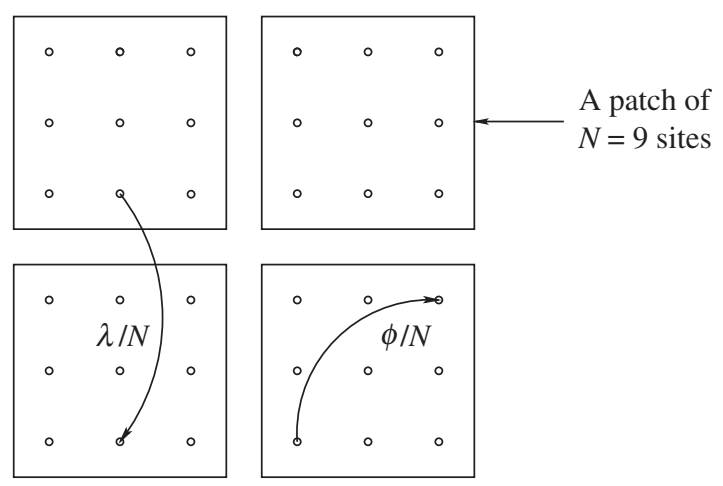

FIgURE 1: The habitat.

$d=2$ ). More precisely, the patches of the habitat are complete graphs $\mathbb{K}_{N}$ with $N$ vertices (that is, any vertex is a neighbor of any other vertex, including itself). In each single patch the sites are enumerated from 1 to $N$ and in each site there might be a priori any number of particles. Formally, the environment is $\mathcal{E}_{N}^{d}:=\mathbb{Z}^{d} \times \mathbb{K}_{N}$ and the state space is $\mathbb{N}_{N}^{d}$. The patch at $x \in \mathbb{Z}^{d}$ is the set $\left\{(x, r): r \in \mathbb{K}_{N}\right\}$. We write $(x, r) \sim\left(y, r_{1}\right)$, meaning that the two sites are in neighboring patches, if and only if $x \sim y$, that is, $|x-y|_{\mathbb{Z}^{d}}=1$ (where $|\cdot|_{\mathbb{Z}^{d}}$ denotes the usual distance in the graph $\mathbb{Z}^{d}$ ).

The dynamics of the process is the following. Each particle dies at rate 1; when a site is vacant, it receives births from each particle in the same patch at rate $\phi / N$ and from each particle in the neighboring patches at rate $\lambda / N$. When a site $x$ is occupied by $i \geq 1$ particles, then it allows birth attempts only from particles in the same patch, but an attempt succeeds only with probability $c(i)$ (the function $c: \mathbb{N} \rightarrow[0,1]$ represents a self-regulating mechanism as in [6]). We suppose that $c(0)=1$ (when a site is vacant, the first reproduction trial is almost surely successful) and that $c$ is nonincreasing (the more individuals present, the less likely it is to produce new offspring). Clearly, if $c(i)=0$ for all $i>i_{0}$ and $c\left(i_{0}\right)>0$, then in each site there might be at most $i_{0}+1$ particles (see Section 2 for the formal definition of the transition rates).

Note that we have two parameters regulating the breeding of a particle: an intra-patch breeding rate $\phi$ and an inter-patch breeding rate being $\lambda$. This kind of biparametric interaction is similar to the one introduced in [11] and later modified in [2]. In both papers the population is divided into equally sized clusters, one cluster per vertex of $\mathbb{Z}^{d}$; in [11] individuals in the same cluster die at the same time, while in [2] they die one at the time. In their model the cluster has no inner structure, that is, the only important attribute is whether it is empty or not (if not, immigration is not allowed). In our model instead of clusters, we have patches and the process depends not only on the total number of particles in the patch, but also on their position among the sites within the patch. For instance, let there be $n$ particles in the patch. If they are on the same site then all sites in the patch but this accept immigration (that is, reproductions from neighboring patches), while intra-patch reproductions are, a priori, always allowed. If they are scattered in $n$ different sites, all these sites will block immigrants.

To strengthen the dependence of the process on the number of individuals per site, we admit a self-regulating mechanism, namely there exists a control function $c \in[0,1]^{\mathbb{N}}$ such that if a site is already occupied by $j$ individuals, newborns are accepted only with probability $c(j)$. This kind of regulating mechanism has been introduced in [6] as a general model of which the 
branching random walk and the contact process are particular cases (when $c \equiv 1$ and $c=\delta_{0}$, respectively). Note that the presence of this function $c$ mimics the fact that when an ecosystem is already exploited by many individuals, the younglings may not have enough food to live and to reach sexual maturity; thus, many of them will not contribute to the breeding process. On the other hand, many animals feed on their younglings if food is scarce.

We observe that the idea of a patchy environment has already been introduced in [3]. The main difference is that in [3] the inter-patch interaction is allowed only between the centers of neighboring patches, while in our model each site may receive immigrants from the neighboring patches.

In the present work we mainly study the effect of the parameters on the probability of survival of the entire population. In particular, we prove that if $\lambda$ and $\phi$ are both small then there is almost-sure extinction (Theorem 3.1). This result is in agreement with the conclusion of the mean-field model in a particular case (see Subsection 2.4). If $\phi$ is small then survival is guaranteed only by $\lambda$ sufficiently large; while large $\phi$ and a 'mild' control function $c$ implies survival even when $\lambda=0$ (the precise statements are Theorem 3.2 and Corollary 3.1, where we give explicit conditions on $\phi$ and $c$ ). Analogously, a large $\lambda$ guarantees survival even when $\phi=0$, while, for small $\lambda$, a large $\phi$ (and a suitable $c$ ) is needed to ensure survival (see Theorem 3.3).

To investigate the impact of different environment structures on the process, we let the patch dimension go to $\infty$. We obtain Theorem 3.4(a), which agrees with the intuition that the limiting process is a branching random walk with intra-site breeding rate $\phi$ and inter-site rate $\lambda$. The reader may want to compare this convergence to the one studied in [8], where a long-range contact process is proved to converge to a branching random walk. We also study the effect of progressively removing the control function: we obtain survival when $\phi>1$ and $c$ is close to 1 (Theorem 3.4(b)). Finally, we study the asymptotic behavior of the critical parameter of the general process and of some particular cases when the size of the patch goes to $\infty$ (Proposition 3.1 and Corollary 3.2) or when the control function is progressively removed (Proposition 3.2).

Here is a brief outline of the paper. In Section 2 we introduce our process and discuss some models that can be seen as particular cases. Subsection 2.4 is devoted to the study of the mean-field model. In Section 3 we discuss our main results; the proofs can be found in Section 4. Section 5 is devoted to the discussion of open questions.

\section{The model}

Given two nonnegative parameters $\phi$ and $\lambda, N \in \mathbb{N}$, and a nonincreasing function $c$ such that $c(0)=1$, the transition rates at $(x, r)$ for the process $\eta_{t}$ are

$$
\begin{aligned}
0 \rightarrow 1 \quad \text { at rate } \frac{\lambda}{N} \sum_{\left\{\left(z, r_{1}\right):\left(z, r_{1}\right) \sim(x, r)\right\}} \eta\left(z, r_{1}\right)+\frac{\phi}{N} \sum_{r_{1} \in \mathbb{K}_{N}} \eta\left(x, r_{1}\right), \\
i \rightarrow i+1 \quad \text { at rate } \frac{\phi}{N} c(\eta(x, r)) \sum_{r_{1} \in \mathbb{K}_{N}} \eta\left(x, r_{1}\right) \text { for } 1 \leq i, \\
i \rightarrow i-1 \quad \text { at rate } i .
\end{aligned}
$$

An equivalent description of the breeding mechanism is that each particle breeds at rate $\phi$ inside its patch and at rate $\lambda$ towards each neighboring patch. Newborns choose the target sites inside the destination patch at random. The reproduction from a different patch is successful only if the target site is vacant, while inside the patch the reproduction is regulated by the function $c$. 
Since we are interested in the survival (with positive probability) or extinction of the population, we always assume that the initial configuration has a finite number of particles. In fact, we observe that the process starting from infinitely many particles never becomes extinct, while the process starting from one initial particle survives with positive probability if and only if the process starting from a finite number of particles survives. Indeed, if it survives starting from one particle, clearly, it survives starting from finitely many particles. Conversely, consider a finite initial condition $\eta_{0}$ and a family of patchy restrained processes (PRPs) $\left\{\tilde{\eta}_{t, i}\right\}_{i=1, \ldots, N}$, where $N=\sum_{x \in \mathcal{E}_{n}^{d}} \eta_{0}(x)$, each one starting from a single particle (the positions are chosen according to the starting vertices of the particles of $\eta_{0}$ ). It is not difficult to prove that the process $\sum_{i=1}^{N} \tilde{\eta}_{t, i}$ dominates the original PRP (starting from $\eta_{0}$ ). Hence, if the original PRP survives then $\sum_{i=1}^{N} \tilde{\eta}_{t, i}$ survives, that is, $\tilde{\eta}_{t, i}$ survives for some $i$. Thus, there is a positive probability of survival starting with a single particle.

In the sequel, we write $\operatorname{PRP}(\lambda, \phi, c, N)$ when we need to stress the dependence on the parameters. We observe that many explicit models are recovered as particular cases of this patchy habitat model.

In particular, by choosing $\phi=0$ we obtain a process which does not depend on $c$ and allows at most one particle per site. We call this process the contact process $(\mathrm{CP})$ on $\varepsilon_{N}^{d}$. It is not difficult to prove that this process has a critical parameter $\lambda_{\mathrm{CP}}\left(\mathcal{E}_{N}^{d}\right) \in\left[1 / 2 d, \lambda_{\mathrm{CP}}\left(\mathbb{Z}^{d}\right)\right]$ (namely the population dies out for $\lambda$ below this value and survives with positive probability for $\lambda$ above this value). Indeed,

$$
\lambda_{\mathrm{CP}}\left(\mathcal{E}_{N}^{d}\right) \leq \lambda_{\mathrm{CP}}\left(\mathcal{E}_{1}^{d}\right)=\lambda_{\mathrm{CP}}\left(\mathbb{Z}^{d}\right)
$$

since $\lambda_{\mathrm{CP}}\left(\varepsilon_{N}^{d}\right)$ is nonincreasing with respect to $N$ and it is possible to prove that $\lambda_{\mathrm{CP}}\left(\varepsilon_{N}^{d}\right) \downarrow 1 / 2 d$ as $N \rightarrow \infty$ (see Proposition 3.1(a)).

On the other hand, if we choose $c=\delta_{0}$, we obtain a process that we call a biparametric contact process $(\mathrm{BCP}(\lambda, \phi))$ on $\varepsilon_{N}^{d}$ which coincides with the contact process when $\phi=0$ (this is in general a process which allows at most one particle per site and has two reproduction rates - the inter-patch $\lambda$ and the intra-patch $\phi$ ).

The individual recovery process (IRP) introduced in [2] can be seen as a particular case of the PRP, namely, by setting $N=1$ and $c=\mathbf{1}_{\{0, \ldots, \kappa-1\}}$ (the patch has only one site, which can host at most $\kappa$ particles).

It is natural to study sequences of PRPs (see Subsection 2.4 and Section 3) by considering a sequence $\left\{N_{n}\right\}_{n \in \mathbb{N}}$ and a corresponding sequence of controlling functions $\boldsymbol{C}=\left\{c_{n}\right\}_{n \in \mathbb{N}}$ (we keep $\lambda$ and $\phi$ fixed). We are interested in the behavior of the process as $n \rightarrow \infty$. We assume that $c_{n} \leq c_{n+1}$ (the regulation gets weaker as $i$ grows). Note that $c_{n}(i)$ may be positive for all $i$; thus, there could be no a priori bounds on the site carrying capacity. We define $c_{\infty}(i):=\lim _{n \rightarrow \infty} c_{n}(i)$ for all $i$.

We conclude this section by listing some particular cases of the PRP which are modifications of the IRP.

\subsection{Logistic IRP}

If we choose $N=1$ and $c(i)=\max \{0,1-i / \kappa\}$ (for some $\kappa \in \mathbb{N} \backslash\{0\}$ ), then we obtain a cluster of size $\kappa$ at each site in $\mathbb{Z}^{d}$ and an interaction between sites which is allowed only when the target is empty. This kind of interaction was introduced by Schinazi [11] with a cluster recovery clearing mechanism (or mass extinction), and modified by Belhadji and Lanchier with individual recoveries in [2]. Note nevertheless that this model differs from the IRP in the fact that the breeding inside the cluster becomes increasingly difficult as we approach the full carrying capacity $\kappa$. For this process $\eta_{t}$, which we call the logistic IRP, the transition rates at 
site $x$ are

$$
\begin{aligned}
& 0 \rightarrow 1 \text { at rate } \lambda \sum_{z \sim x} \eta(z), \\
& i \rightarrow i+1 \quad \text { at rate } i \phi\left(1-\frac{i}{\kappa}\right) \text { for } 1 \leq i \leq \kappa-1, \\
& i \rightarrow i-1 \quad \text { at rate } i \text { for } i \geq 1 .
\end{aligned}
$$

Clearly, the presence of the logistic factor $1-i / \kappa$ in the breeding rate may be interpreted as a self-regulating mechanism of the population which slows down the reproductions when the patch is almost completely exploited.

\subsection{Self-regulating IRP}

If $N=1$, in general we obtain a process $\eta_{t}$ that we call a self-regulating IRP. The process has the following rates at site $x$ :

$$
\begin{aligned}
& 0 \rightarrow 1 \quad \text { at rate } \lambda \sum_{z \sim x} \eta(z), \\
& i \rightarrow i+1 \quad \text { at rate } i \phi c(i), \\
& i \rightarrow i-1 \quad \text { at rate } i .
\end{aligned}
$$

\subsection{Logistic IRP with persistent inter-patches reproduction}

Given a general PRP, we may identify all the sites in the same patch, meaning that we consider the process $\xi_{t}(x)=\sum_{r \in \mathbb{K}_{N}} \eta_{t}(x, r)$. We refer to $\xi_{t}$ as the projection on $\mathbb{Z}^{d}$ of $\eta_{t}$. The projection is a Markov process only when $c \equiv \delta_{0}$. In this case it has the following transition rates at site $x$ :

$$
\begin{aligned}
& i \rightarrow i+1 \quad \text { at rate }\left(i \phi+\lambda \sum_{z \sim x} \xi(z)\right)\left(1-\frac{i}{N}\right) \text { for } 0 \leq i \leq N-1, \\
& i \rightarrow i-1 \text { at rate } i \text { for } i \geq 1
\end{aligned}
$$

We note that this process is similar to the IRP of [2] with cluster size equal to $N$. Nevertheless, it has two main differences: the interaction between clusters is always active (while in the IRP it vanishes once the cluster is nonempty) and it gets more difficult to increase the number of individuals in the cluster if the cluster is crowded.

\subsection{The mean-field equations for the self-regulating IRP}

The usual approach, before actually studying the spatial stochastic model, is to derive a nonspatial deterministic version called the mean field. We consider the differential evolution equations for the concentrations $\left\{u_{i}\right\}_{i \geq 0}$, where $u_{i}=u_{i}(t)$ can be thought of as the proportion of sites with $i$ individuals at time $t$ when there is a very large population (clearly, $\sum_{i} u_{i}=1$ ). We compute the stationary solutions of the system of equations: a stationary solution with $u_{0}<1$ corresponds to survival, while $u_{0} \geq 1$ suggests almost-sure extinction. To avoid unnecessary complications, in this preliminary study, we take $N=1$, that is, there is no difference between patches and sites. 
Let us briefly discuss the mean-field equation for the logistic IRP. The mean-field equations are

$$
\begin{aligned}
& u_{0}^{\prime}=u_{1}-\lambda u_{0} \sum_{i=1}^{\kappa} i u_{i}, \\
& u_{1}^{\prime}=2 u_{2}+\lambda u_{0} \sum_{i=1}^{\kappa} i u_{i}-u_{1}\left(1+\phi\left(1-\frac{1}{\kappa}\right)\right), \\
& u_{i}^{\prime}=(i+1) u_{i+1}+(i-1)\left(1-\frac{i-1}{\kappa}\right) \phi u_{i-1}-i\left(1+\left(1-\frac{i}{\kappa}\right) \phi\right) u_{i}, \quad 1 \leq i \leq \kappa-1, \\
& u_{\kappa}^{\prime}=-\kappa u_{\kappa}+(\kappa-1)\left(1-\frac{\kappa-1}{\kappa}\right) \phi u_{\kappa-1} .
\end{aligned}
$$

Set $u_{i}^{\prime}=0$ for $i=1, \ldots, \kappa$ and sum the equations from $i$ to $\kappa$. Then the solution, for $i=1, \ldots, \kappa$, is

$$
u_{i}=\frac{(\kappa-1) !}{(\kappa-i) ! i}\left(\frac{\phi}{\kappa}\right)^{i-1} u_{1} .
$$

We substitute this into the first equation and require that $u_{0}^{\prime}=0$ to obtain

$$
u_{0}=\frac{1}{\lambda}\left(\sum_{i=0}^{\kappa-1} \frac{(\kappa-1) !}{(\kappa-i-1) !}\left(\frac{\phi}{\kappa}\right)^{i}\right)^{-1}=\frac{1}{\lambda}\left(1+\sum_{i=1}^{\kappa-1} \phi^{i} \prod_{j=1}^{i}\left(1-\frac{j}{\kappa}\right)\right)^{-1} .
$$

From this we see that $u_{0} \rightarrow 0$ as $\kappa \rightarrow \infty$ (thus indicating the possibility of an endemic state for large $\kappa)$ when $\phi \geq 1(\phi>1$ implies exponential convergence, and when $\phi=1$, then $\left.u_{0} \sim C / \lambda \sqrt{\kappa}\right)$.

More precisely, the monotone convergence theorem implies that $u_{0} \downarrow \min (0,(1-\phi) / \lambda)$ as $\kappa \rightarrow \infty$. Hence, if $\phi+\lambda \leq 1$ then $u_{0} \geq 1$ for all $\kappa$, while if $\phi+\lambda>1$ then $u_{0}<1$ for sufficiently large $\kappa$.

If we consider the self-regulating IRP, the mean-field equations are

$$
\begin{aligned}
& u_{0}^{\prime}=u_{1}-\lambda u_{0} \sum_{i=1}^{\infty} i u_{i}, \\
& u_{1}^{\prime}=2 u_{2}+\lambda u_{0} \sum_{i=1}^{\infty} i u_{i}-u_{1}(1+\phi c(1)), \\
& u_{i}^{\prime}=(i+1) u_{i+1}+(i-1) c(i-1) \phi u_{i-1}-i u_{i}(1+\phi c(i)), \quad 1 \leq i .
\end{aligned}
$$

We can write the solution of the system $u_{i}^{\prime}=0$ for $i \geq 1$ as

$$
u_{i}=\frac{\phi^{i-1}}{i} \prod_{l=0}^{i-1} c(l) u_{1}
$$

We substitute this into the first equation and require that $u_{0}^{\prime}=0$ to obtain

$$
u_{0}=\frac{1}{\lambda \sum_{i=0}^{\infty} \phi^{i} \prod_{l=0}^{i} c(l)} .
$$


If we are given a sequence of processes regulated by the functions $\left\{c_{n}\right\}_{n \geq 0}$, since $c_{n} \uparrow c_{\infty}$ as $n \rightarrow \infty$, by the monotone convergence theorem we have

$$
u_{0}=\frac{1}{\lambda \sum_{i=0}^{\infty} \phi^{i} \prod_{l=0}^{i} c_{n}(l)} \downarrow \frac{1}{\lambda \sum_{i=0}^{\infty} \phi^{i} \prod_{l=0}^{i} c_{\infty}(l)} \geq \min \left(0, \frac{1-\phi}{\lambda}\right),
$$

and the equality holds if and only if $c_{\infty} \equiv 1$. In this case, as before for the logistic IRP, $\phi+\lambda \leq 1$ implies that $u_{0} \geq 1$ for all $c_{n}$, while $\phi+\lambda>1$ implies that $u_{0}<1$ for sufficiently large $n$.

The mean-field model for the self-regulating IRP thus suggests that, in the spatial case, if $\phi+2 d \lambda \leq 1$, there is extinction for all controlling functions $c_{n}$ (see Theorem 3.1) and, if $c_{\infty} \equiv 1$, survival is implied by $\phi+2 d \lambda>1$ provided that $n$ is sufficiently large (we prove a slightly different result; see Theorem 3.4).

\section{Main results and discussion}

In this section we discuss the effect of the parameters $\lambda, \phi, c$, and $N$ on the behavior of the PRP. Parts of the argument are done using monotonicity and coupling with known processes. Indeed, for any fixed $N$, the PRP is attractive with respect to $\lambda, \phi$, and $c$. Moreover, we may couple a PRP on $\varepsilon_{N}^{d}$ with another PRP on $\varepsilon_{1}^{d} \equiv \mathbb{Z}^{d}$ in the following natural way. Consider two PRPs: $\left\{\eta_{t}\right\}_{t \geq 0}$ on $\mathcal{E}_{N}^{d}$ (with parameters $\lambda, \phi$ and function $c$ ) and $\left\{\xi_{t}\right\}_{t \geq 0}$ on $\mathbb{Z}^{d}$ (with parameters $\lambda_{1}, \phi_{1}$, and function $c_{1}$ ). If $\lambda \geq \lambda_{1}, \phi \geq \phi_{1}$, and $c \geq c_{1}$, then the projection of $\eta_{t}$ on $\mathbb{Z}^{d}$ (namely $\sum_{r \in \mathbb{K}_{N}} \eta_{t}(\cdot, r)$ ) dominates $\xi_{t}(\cdot)$.

This kind of coupling is the key to the proof of the following result, which states that if the breeding parameters $\lambda$ and $\phi$ are sufficiently small then we have almost-sure extinction.

Theorem 3.1. For all functions $c$, if $\phi+2 d \lambda \leq 1$ then there is almost-sure extinction.

By monotonicity, it is clear that, if $N, c$, and $\phi$ are fixed then there exists a critical $\lambda_{\mathrm{c}}=$ $\lambda_{\mathrm{c}}(\phi, c, N)$ such that, given $\lambda>\lambda_{\mathrm{c}}$, the PRP survives with positive probability, while $\lambda<\lambda_{\mathrm{c}}$ implies almost-sure extinction. By stochastic domination, $\lambda_{\mathrm{c}} \leq \lambda_{\mathrm{CP}}\left(\mathcal{E}_{N}^{d}\right)$; thus, if $\lambda>\lambda_{\mathrm{CP}}\left(\varepsilon_{N}^{d}\right)$ then the PRP survives with positive probability for all choices of $\phi$ and $c$.

The following theorem describes the dependence of $\lambda_{c}$ on $\phi$ and $c$. Since $c(i)$ can be equal to 0 for some $i$, we identify $1 / 0$ with $+\infty$.

Theorem 3.2. (a) Given any $\phi$ and $c$ such that $\sum_{n=0}^{\infty}\left(\phi^{n} \prod_{i=0}^{n} c(i)\right)^{-1}<+\infty$, then, for every $\lambda \geq 0$, the PRP survives with positive probability; hence, $\lambda_{\mathrm{c}}(\phi, c, N)=0$.

(b) Given any $\phi$ and $c$ such that $\sum_{n=0}^{\infty} \phi^{n} \prod_{i=0}^{n} c(i)<+\infty$, then $\lambda_{\mathrm{c}}(\phi, c, N) \in\left(0, \lambda_{\mathrm{CP}}\left(\varepsilon_{N}^{d}\right)\right)$.

In particular, from the previous theorem we deduce that the value of $\lim _{i \rightarrow \infty} c(i$ ) (which exists by the assumption of monotonicity that we made) tells us whether $\lambda_{c}=0$ or not in almost every case.

Corollary 3.1. Let $c(\infty)=\lim _{i \rightarrow \infty} c(i)$. If $\phi c(\infty)>1$ then $\lambda_{\mathrm{c}}(\phi, c, N)=0$. If $\phi c(\infty)<1$ then $\lambda_{\mathrm{c}}(\phi, c, N)>0$. As a particular case, for all functions $c, \phi<1$ implies that $\lambda_{\mathrm{c}}>0$.

In the case $\phi c(\infty)=1$ the behavior depends on the speed of convergence of $c(n)$ to $c(\infty)$. For instance, if $c(n):=(n+3)^{2} / 2(n+2)^{2}$ and $\phi=2$, then Theorem 3.2(a) applies and $\lambda_{\mathrm{c}}=0$. Unfortunately, due to the monotonicity of $c$, in the case $\phi c(\infty)=1$, Theorem 3.2(b) is useless (since the series is always divergent). 
Again, by monotonicity, if $N, c$, and $\lambda$ are fixed then there exists a critical $\phi_{\mathrm{c}}=\phi_{\mathrm{c}}(\lambda, c, N)$ such that, given $\phi>\phi_{\mathrm{c}}$, the PRP survives with positive probability, while $\phi<\phi_{\mathrm{c}}$ implies almost-sure extinction. In general, it could happen that $\phi_{\mathrm{c}}=\infty$ (almost-sure extinction for all $\phi$-for instance, when $N=1, c=\delta_{0}$, and $\lambda<\lambda_{\mathrm{CP}}\left(\mathbb{Z}^{d}\right)$ ) or $\phi_{\mathrm{c}}=0$ (survival for all positive $\phi$-for instance, when $\left.\lambda>\lambda_{\mathrm{CP}}\left(\mathcal{E}_{N}^{d}\right)\right)$. The following theorem gives sufficient conditions for $\phi_{\mathrm{c}} \in(0, \infty)$.

Theorem 3.3. (a) If $\lambda>\lambda_{\mathrm{CP}}\left(\varepsilon_{N}^{d}\right)$ then $\phi_{\mathrm{c}}(\lambda, c, N)=0$ for all $c$ and $N$, and the PRP survives with positive probability when $\phi=0$.

(b) For any $\lambda \in(0,1 / 2 d)$ and $c$ such that $c(1)>0$, then $\phi_{\mathrm{c}}(\lambda, c, N) \in(0,+\infty)$.

Actually, to prove that $\phi_{\mathrm{c}}<\infty$, we only need the hypothesis $c(1)>0$, while $\lambda \in(0,1 / 2 d)$ is needed to prove that $\phi_{\mathrm{c}}>0$. We guess that $\phi_{\mathrm{c}}>0$ could be proved under the milder assumption that $\lambda \in\left(0, \lambda_{\mathrm{CP}}\left(\varepsilon_{N}^{d}\right)\right)$; in order to do so, we could adapt the proofs of [2, Theorem 3] and [11, Theorem 1(c)]. The main difficulty is to prove the analog of [7, Theorem 1.7] for the CP on $\varepsilon_{N}^{d}$. Since $\lambda_{\mathrm{CP}}\left(\varepsilon_{N}^{d}\right) \downarrow 1 / 2 d$, extending the interval for $\lambda$ to $\left(0, \lambda_{\mathrm{CP}}\left(\varepsilon_{N}^{d}\right)\right)$ seems to be a minor improvement at least for large values of $N$.

Now we want to study the dependence of the behavior of the PRP on the underlying space: note that 'space' here is described both by $N$ (the horizontal space) and $c$ (the vertical space). Hence, it is natural to define a sequence of PRPs by means of nondecreasing sequences of functions $\left\{c_{n}\right\}_{n \in \mathbb{N}}$ and of patch dimensions $\left\{N_{n}\right\}_{n \in \mathbb{N}}$. More precisely, the $i$ th process is $\mathbb{N}^{\varepsilon_{N_{n}}^{d}}$ valued and its transition rates are defined by (2.1a)-(2.1c) with $c=c_{n}(d, \lambda$, and $\phi$ being fixed for all $n$ ).

Theorem 3.4. Consider a sequence of PRPs on $\mathcal{E}_{N_{n}}^{d}$ with parameters $\phi$ and $\lambda$, and control functions $c_{n}$. If $\lambda>0$ and either

(a) $\phi+2 d \lambda>1$ and $N_{n} \rightarrow \infty$; or

(b) $\phi \inf _{i \in \mathbb{N}} c_{\infty}(i)>1$;

holds, then there exists $n_{\mathrm{c}}=n_{\mathrm{c}}(\lambda, \phi, C)$ such that, for all $n \geq n_{\mathrm{c}}$, there is survival with positive probability.

Roughly speaking, Theorem 3.4 states that if the space is sufficiently large then the PRP survives with positive probability provided that the breeding parameters are not too small. In particular, Theorem 3.4(a) is a partial converse of Theorem 3.1. In the proof we need to mimic a technique introduced in [4] in order to show the 'convergence' of the projections on $\mathbb{Z}^{d}$ of the PRPs to a branching random walk.

Remark 3.1. Theorems 3.1 and 3.4 imply, roughly speaking, that, when $N_{n} \rightarrow \infty$, the projection of the $n$th PRP on $\mathbb{Z}^{d}$ behaves, in the limit $n \rightarrow \infty$, as the branching random walk on $\mathbb{Z}^{d}$ with intra-site breeding rate $\phi$ and inter-site rate $\lambda$, in the sense that it survives eventually if and only if $\phi+2 d \lambda>1$.

Recall that, by [2, Theorem 5] for the IRP with parameters $\lambda$ and $\phi$ and with $\kappa$ maximal number of particles per vertex, there is a critical value for $\kappa$, say $\kappa_{\mathrm{c}}(\lambda, \phi)$, such that, for $\kappa \geq \kappa_{\mathrm{c}}(\lambda, \phi)$, there is survival and, for $\kappa<\kappa_{\mathrm{c}}(\lambda, \phi)$, there is extinction. It is easy to prove that condition (b) of Theorem 3.4 can be relaxed, provided that we have some knowledge of the function $\kappa_{\mathrm{c}}$. More precisely, we can prove (using the same coupling arguments as in the proof of Theorem 3.4) that if, given $\lambda>0, \phi \geq 0$, and $\left\{c_{n}\right\}$, there exists $\tilde{\phi}>1$ such that 
$\phi c_{\infty}\left(\kappa_{\mathrm{c}}(\lambda, \tilde{\phi})-1\right)>\tilde{\phi}$ then there exists $n_{\mathrm{c}}$ such that, for all $n \geq n_{\mathrm{c}}$, there is survival with positive probability.

The following proposition deals with the asymptotic behavior of the critical parameters of various processes on $\varepsilon_{N_{n}}^{d}$ as $N_{n} \rightarrow \infty$. The results follow from Theorems 3.1 and 3.4.

Proposition 3.1. If $N_{n} \uparrow \infty$ the following statements hold.

(a) $\lambda_{\mathrm{CP}}\left(\mathcal{E}_{N_{n}}^{d}\right) \downarrow 1 / 2 d$.

(b) Consider the biparametric contact process. For all fixed $\phi \geq 0$, we have

$$
\lambda_{\mathrm{BCP}}\left(\mathcal{E}_{N_{n}}^{d}, \phi\right) \downarrow \max \left(\frac{1-\phi}{2 d}, 0\right),
$$

while, for all fixed $\lambda>0$, we have

$$
\phi_{\mathrm{BCP}}\left(\mathcal{E}_{N_{n}}^{d}, \lambda\right) \downarrow \max (1-2 d \lambda, 0) .
$$

In the particular case in which $\phi=\lambda$ (the intra-patch reproduction has the same rate as the inter-patch reproduction), the critical parameter converges to $1 /(2 d+1)$.

(c) For a sequence of PRPs,

$$
\begin{gathered}
\lim _{n \rightarrow \infty} \lambda_{\mathrm{c}}\left(\phi, c_{n}, N_{n}\right)=0 \quad \text { if } \phi>1, \\
\lim _{n \rightarrow \infty} \phi_{\mathrm{c}}\left(\lambda, c_{n}, N_{n}\right)=0 \quad \text { if } \lambda>1 / 2 d .
\end{gathered}
$$

We note that Proposition 3.1(c) gives the limit of $\lambda_{\mathrm{c}}$ as the size of the patch goes to $\infty$. The following proposition deals with the limit of $\lambda_{\mathrm{c}}$ in the case in which $c_{\infty} \equiv 1$ ( $N$ and $\phi$ being fixed). It is a consequence of Corollary 3.1 and Theorem 3.4(b), and establishes the continuity of $\lambda_{\mathrm{c}}$ with respect to $c$.

Proposition 3.2. Given $N \in \mathbb{N}, \phi>1$, and a sequence of functions $\left\{c_{n}\right\}_{n \geq 0}$ such that $c_{\infty} \equiv 1$, then

$$
\lim _{n \rightarrow \infty} \lambda_{\mathrm{c}}\left(\phi, c_{n}, N\right)=\lambda_{\mathrm{c}}\left(\phi, c_{\infty}, N\right) .
$$

We recall that the IRP with cluster size $\kappa$ is a particular case of the PRP (taking $N=1$ and $\left.c=\mathbf{1}_{\{0, \ldots, \kappa-1\}}\right)$. Thus, if $\bar{\lambda}^{\kappa}(\phi)$ is the critical parameter of this IRP, from Proposition 3.2 we obtain the following result on the asymptotic behavior of $\bar{\lambda}^{\kappa}(\phi)$ as $\kappa$ goes to $\infty$.

Corollary 3.2. If $\phi>1$ then $\lim _{\kappa \rightarrow \infty} \bar{\lambda}^{\kappa}(\phi)=0$.

We note that, if $\bar{\lambda}^{\infty}(\phi)$ is the critical parameter of the IRP with cluster size equal to $\infty$ (see [1] for some details on this process) then, as a consequence of Corollary 3.1, if $\phi>1$ then $\bar{\lambda} \infty(\phi)=0$. Thus, the previous corollary is a result of continuity at $\infty$ for $\bar{\lambda}^{\kappa}(\phi)$ with respect to the cluster size.

\section{Proofs}

Proof of Theorem 3.1. It is enough to note that the total reproduction rate of each particle is bounded from above by $\phi+2 d \lambda$. Indeed, the projection $\sum_{k \in \mathbb{K}_{n}} \tilde{\eta}_{t}(x, k)$ is dominated by a branching random walk on $\mathbb{Z}^{d}$ with intra-patch infection rate $\phi$ and inter-patch rate $\lambda$ on each edge. In this branching random walk each particle breeds at rate $\phi+2 d \lambda$ and dies at rate 1 .

In order to prove Theorem 3.2, we need the following lemma on discrete-time random walks. 
Lemma 4.1. Let $\left\{Z_{n}\right\}_{n \geq 0}$ be a random walk on $\mathbb{N}^{N}$ with the following transition probabilities:

$$
\begin{aligned}
& \left(i_{1}, \ldots, i_{N}\right) \rightarrow\left(i_{1}, \ldots, i_{j}+1, \ldots, i_{N}\right) \quad \text { with probability } \frac{\phi c\left(i_{j}\right)}{(1+\phi) N}, \\
& \left(i_{1}, \ldots, i_{N}\right) \rightarrow\left(i_{1}, \ldots, i_{j}-1, \ldots, i_{N}\right) \quad \text { with probability } \frac{1}{(1+\phi) N}, \\
& \left(i_{1}, \ldots, i_{N}\right) \rightarrow\left(i_{1}, \ldots, i_{N}\right) \quad \text { with probability } \frac{\phi}{(1+\phi) N} \sum_{j=1}^{N}\left(1-c\left(i_{j}\right)\right) .
\end{aligned}
$$

(a) If $\sum_{n=0}^{\infty}\left(\phi^{n} \prod_{i=0}^{n} c(i)\right)^{-1}<+\infty$ then the random walk is transient.

(b) The random walk is positive recurrent if and only if $\sum_{n=0}^{\infty} \phi^{n} \prod_{i=0}^{n} c(i)<+\infty$.

Proof. (a) We note that the projection of the $N$-dimensional process on the $j$ th coordinate is a birth-and-death process with forward probabilities $\phi c(i) /(N(1+\phi))$ and backward probabilities $1 /(N(1+\phi))$, which, by [13, Theorem 5.9], is transient if and only if $\sum_{n=0}^{\infty}\left(\phi^{n} \prod_{i=0}^{n} c(i)\right)^{-1}<+\infty$. But transience of at least one projection implies transience of the $N$-dimensional process.

(b) Positive recurrence is equivalent to the existence of a finite invariant measure. It is not difficult to prove that

$$
v\left(i_{1}, \ldots, i_{N}\right)=\prod_{\left\{j: i_{j}>0\right\}}\left(\phi^{i_{j}} \prod_{i=0}^{i_{j}-1} c(i)\right), \quad v(0, \ldots, 0)=1,
$$

is a reversible measure. The claim follows noting that

$$
v\left(\mathbb{N}^{N}\right)=\sum_{i_{1}, \ldots, i_{N}} v\left(i_{1}, \ldots, i_{N}\right)=\left(1+\phi \sum_{n=0}^{+\infty} \phi^{n} \prod_{i=0}^{n} c(i)\right)^{N} .
$$

Proof of Theorem 3.2. (a) Take the PRP with $\lambda=0$. Since it cannot leave the original patch, this is a (continuous-time) random walk on $\mathbb{N}^{N}$ and it is stochastically dominated by the original PRP; in this case survival means 'avoiding the origin'. The survival of the (continuoustime) random walk is equivalent to the survival of its discrete counterpart (see (4.1)) which is guaranteed by Lemma 4.1(a) noting that transience implies survival.

(b) By (a), $\lambda_{\mathrm{c}} \leq \lambda_{\mathrm{CP}}\left(\varepsilon_{N}^{d}\right)$. We now prove that $\lambda_{\mathrm{c}}>0$. Let us start by considering the case in which $N=1$ (remember that $\varepsilon_{1}^{d}$ can be identified with $\mathbb{Z}^{d}$ ). For small $\lambda$, we want to find a subcritical branching process which dominates the total number of successful inter-patch reproductions. Indeed, in this case the PRP cannot survive without leaving the first patch; hence, survival is equivalent to the positive probability of having an infinite number of successful interpatch infections. Once a particle appears at $x$, the total number of particles living at $x$ up to the next time when the colony at $x$ has no individuals is, according to Lemma 4.1, a positive recurrent random walk (let us call $\tau_{0}$ the number of steps before reaching 0 ) with the following rates:

$i \rightarrow i+1 \quad$ with rate $i \phi c(i), \quad i \rightarrow i-1 \quad$ with rate $1, \quad i \rightarrow i \quad$ with rate $i \phi(1-c(i))$.

Note that the exponential clock of the transition (including the loop) has parameter $i(1+\phi)$. 
The number of inter-patch reproduction trials made from site $x$ depends on the number of particles at $x$ : indeed, when the number of particles at $x$ is $i$, the total reproduction rate towards the neighboring patches is $i \lambda$. Hence, the number of inter-patch reproduction trials between two transitions does not depend on $i$, since it is the number of times a Poisson clock with rate $i \lambda$ rings before the transition clock with rate $i(1+\phi)$ rings. It is clear that the total number of reproduction trials (before the progeny of the original particle at $x$ dies out) from $x$ to the neighbors is $\sum_{k=1}^{\tau_{0}} \sum_{j=1}^{2 d} Y_{k}(j)$, where $\left\{Y_{k}\right\}_{k \geq 1}$ is an independent and identically distributed sequence of random $2 d$-dimensional vectors; to be precise, if $n_{i}$ is the number of reproduction trials on neighbor $i$ then

$$
\mathrm{P}\left(Y_{k}=\left(n_{1}, \ldots, n_{2 d}\right)\right)=\frac{\left(\sum_{i=1}^{2 d} n_{i}\right) !(\lambda /(1+\phi))^{\sum_{i=1}^{2 d} n_{i}}}{(1+2 d \lambda /(1+\phi))^{1+\sum_{i=1}^{2 d} n_{i}} \prod_{i=1}^{2 d} n_{i} !}
$$

(see [5] for details). Thus,

$$
\mathrm{E}\left(\sum_{k=1}^{\tau_{0}} \sum_{j=1}^{2 d} Y_{k}(j)\right)=2 d \frac{\lambda}{1+\phi} \mathrm{E}\left(\tau_{0}\right) \rightarrow 0
$$

as $\lambda \rightarrow 0$. By choosing $\lambda<(1+\phi) /\left(2 d \mathrm{E}\left(\tau_{0}\right)\right)$, the total number of successful trials (which cannot exceed the total number of trials) is dominated by a (subcritical) branching process with expected number of offspring $2 d \lambda \mathrm{E}\left(\tau_{0}\right) /(1+\phi)<1$.

Let us take $N>1$ : we cannot repeat the same argument exactly since generations inside a patch are not independent, due to the presence of the controlling function $c$. Hence, we couple with a suitable process. The coupled process has the following rules:

(i) every inter-patch reproduction is successful (i.e. we do not use $c$ );

(ii) we define the particle born from an inter-patch reproduction as the ancestor of its descendants inside its patch;

(iii) inside a patch particles compete for resources if and only if they have the same ancestor (i.e. the function $c$ applies inside the same progeny).

Clearly, this process dominates the original PRP and progenies of different ancestors are independent.

The number of generations of the progeny of a particle in the dominating process is equal to the absorption time $\tau_{0}$ of a random walk with rates

$$
\begin{aligned}
& \left(i_{1}, \ldots, i_{N}\right) \rightarrow\left(i_{1}, \ldots, i_{j}+1, \ldots, i_{N}\right) \quad \text { with rate } \phi c\left(i_{j}\right), \\
& \left(i_{1}, \ldots, i_{N}\right) \rightarrow\left(i_{1}, \ldots, i_{j}-1, \ldots, i_{N}\right) \quad \text { with rate } 1 \\
& \left(i_{1}, \ldots, i_{N}\right) \rightarrow\left(i_{1}, \ldots, i_{N}\right) \quad \text { with rate } \phi \sum_{j=1}^{N}\left(1-c\left(i_{j}\right)\right) .
\end{aligned}
$$

As before, the behavior of this random walk is equivalent to the behavior of its discrete-time counterpart which, according to Lemma 4.1, is positive recurrent (thus, $\mathrm{E}\left(\tau_{0}\right)<+\infty$ ). Since the discrete-time counterpart is recurrent, then survival is equivalent to the positive probability of having an infinite number of progenies. But the expected number of successful inter-patch reproductions is equal to $2 d \lambda \mathrm{E}\left(\tau_{0}\right) /(1+\phi)$. Choosing small enough $\lambda$ yields the conclusion. 
Proof of Theorem 3.3. (a) It follows from the fact that, for all $\phi \geq 0$, the PRP stochastically dominates the $\mathrm{CP}$ on $\varepsilon_{N}^{d}$.

(b) The hypothesis $c(1)>0$ yields $\phi_{\mathrm{c}}<\infty$. Indeed, let $\tilde{\phi}_{\mathrm{c}}(\lambda, 2)>0$ be the critical threshold in [2, Theorem 3] and choose $\phi>\tilde{\phi}_{\mathrm{c}}(\lambda, 2) / c(1)$. Let $\tilde{\eta}_{t}: \mathbb{Z}^{d} \rightarrow\{0,1,2\}$ be the IRP with parameters $\widetilde{\lambda}=\lambda, \widetilde{\phi}=\phi c(1)$, and $\widetilde{\kappa}=2$. By these choices of parameters, $\tilde{\eta}_{t}$ is supercritical. On the other hand, the projection $\xi_{t}(x)=\sum_{i \in \mathbb{K}_{n}} \tilde{\eta}_{t}(x, i)$ dominates $\tilde{\eta}_{t}$ and this proves that the PRP survives when $\phi>\tilde{\phi}_{\mathrm{c}}(\lambda, 2) / c(1)$.

To show that $\phi_{\mathrm{c}}>0$ in the case where $\lambda<1 / 2 d$, note that, by Theorem 3.1, if we choose a positive $\phi$ smaller than $1-2 d \lambda$ then the population dies out.

Proof of Theorem 3.4. (a) The PRPs of the sequence dominate the PRPs where the control functions are equal to $\delta_{0}$ for all $n$. Hence, it is enough to prove the statement for the latter case.

The projection of this process on $\mathbb{Z}^{d}$, namely $\xi_{t}^{n}(x):=\sum_{r \in \mathbb{K}_{N}} \eta_{t}^{n}(x, r)$, has transition rates, at $x \in \mathbb{Z}^{d}$,

$$
\begin{aligned}
& j \rightarrow j+1 \quad \text { at rate }\left(j \phi+\lambda \sum_{y \sim x} \xi_{t}^{n}(y)\right)\left(1-\frac{j}{N_{n}}\right), \\
& j \rightarrow j-1 \quad \text { at rate } j .
\end{aligned}
$$

This process dominates eventually (as $N_{n} \rightarrow \infty$ ) the process $\left\{\tilde{\xi}_{t}\right\}_{t \geq 0}$ with rates

$$
\begin{aligned}
& j \rightarrow j+1 \quad \text { at rate }\left(j \phi+\lambda \sum_{y \sim x} \tilde{\xi}_{t}(y)\right)(1-\varepsilon) \mathbf{1}_{[0, \bar{N}]}(j), \\
& j \rightarrow j-1 \text { at rate } j
\end{aligned}
$$

for all $\bar{N}>0$ and $\varepsilon>0$ (it suffices that $\left.N_{n}>\bar{N} / \varepsilon\right)$. Choose $\varepsilon>0$ such that $(\phi+2 d \lambda)(1-\varepsilon)>1$. We will now prove, following [4], that, for all sufficiently large $\bar{N}$, the process $\left\{\tilde{\xi}_{t}\right\}_{t \geq 0}$ survives with positive probability. The strategy is to study the branching random walk $\left\{\bar{\xi}_{t}\right\}_{t \geq 0}$ with rates

$$
\begin{aligned}
& j \rightarrow j+1 \quad \text { at rate }\left(j \bar{\phi}+\bar{\lambda} \sum_{y \sim x} \bar{\xi}_{t}(y)\right), \\
& j \rightarrow j-1 \quad \text { at rate } j,
\end{aligned}
$$

starting with one particle at the origin $0 \in \mathbb{Z}^{d}$, where $\bar{\phi}=(1-\varepsilon) \phi$ and $\bar{\lambda}=(1-\varepsilon) \lambda$. Note that the process $\left\{\tilde{\xi}_{t}\right\}_{t \geq 0}$ can be seen as a truncation (at height $\bar{N}$ ) of $\left\{\bar{\xi}_{t}\right\}_{t \geq 0}$.

It is not difficult to verify that $\mathrm{E}^{\delta_{0}}\left(\bar{\xi}_{t}\right)$ satisfies the following differential equation (see [4, Section 5], where we did the same with the classical branching random walk):

$$
\frac{\mathrm{d}}{\mathrm{d} t} \mathrm{E}^{\delta_{0}}\left(\bar{\xi}_{t}(x)\right)=-\mathrm{E}^{\delta_{0}}\left(\bar{\xi}_{t}(x)\right)+\phi \mathrm{E}^{\delta_{0}}\left(\bar{\xi}_{t}(x)\right)+\lambda \sum_{y \sim x, y \neq x} \mathrm{E}^{\delta_{0}}\left(\bar{\xi}_{t}(y)\right),
$$

whose solution is

$$
\mathrm{E}^{\delta_{0}}\left(\bar{\xi}_{t}(x)\right)=\sum_{n=0}^{\infty} \sum_{k=0}^{n-|x|_{\mathbb{Z}^{d}}} \mu^{(n, k)}(0, x) \frac{\bar{\phi}^{k} \bar{\lambda}^{n-k} t^{n}}{n !} \mathrm{e}^{-t},
$$

where $\mu^{(n, k)}(0, x)$ is the number of paths from 0 to $x$ of length $n$ and $k$ loops. Moreover, if 
$|x|_{\mathbb{Z}^{d}}=1$ then, taking $t=n$ large enough,

$$
\begin{aligned}
\mathrm{E}^{\delta_{0}}\left(\bar{\xi}_{t}(x)\right) & \geq \sum_{k=0}^{n-1} \mu^{(n, k)}(0, x) \frac{\bar{\phi}^{k} \bar{\lambda}^{n-k} t^{n}}{n !} \mathrm{e}^{-t} \\
& =\sum_{k=0}^{n-1} \mu^{(n, k)}(0, x) \frac{\bar{\phi}^{k} \bar{\lambda}^{n-k}}{(\bar{\phi}+2 d \bar{\lambda})^{n}} \frac{n^{n}(\bar{\phi}+2 d \bar{\lambda})^{n}}{n !} \mathrm{e}^{-n} \\
& \stackrel{\sim}{\sim} \frac{(\bar{\phi}+2 d \bar{\lambda})^{n}}{\sqrt{2 \pi n}} \sum_{k=0}^{n-1} \mu^{(n, k)}(0, x) \frac{\bar{\phi}^{k} \bar{\lambda}^{n-k}}{(\bar{\phi}+2 d \bar{\lambda})^{n}} \\
& \geq \frac{(\bar{\phi}+2 d \bar{\lambda})^{n}}{\sqrt{2 \pi n}} C n^{-d / 2}
\end{aligned}
$$

for some $C=C(\bar{\lambda}, \bar{\phi})>0$, since $\sum_{k=0}^{n-1} \mu^{(n, k)}(0, x) \bar{\phi}^{k} \bar{\lambda}^{n-k} /(\bar{\phi}+2 d \bar{\lambda})^{n}$ is the probability of being in $x\left(|x|_{\mathbb{Z}^{d}}=1\right)$ after $n$ steps for a discrete-time random walk with transition probabilities

$$
p(x, y)= \begin{cases}\frac{\bar{\lambda}}{\bar{\phi}+2 d \bar{\lambda}} & \text { if } x \sim y, \\ \frac{\bar{\phi}}{\bar{\phi}+2 d \bar{\lambda}} & \text { if } x=y\end{cases}
$$

(see [12, Corollary 13.11] for the asymptotic estimates of the $n$-step probabilities). Hence, there exists $t=n$ sufficiently large such that $\mathrm{E}^{\delta_{0}}\left(\bar{\xi}_{t}(x)\right)>1$ (this result is analogous to Lemmas 5.2 and 5.3 of [4]). Using the same arguments as in Lemma 5.4, Remark 5.5, and Theorem 5.6 of [4], it is possible to prove that the process $\left\{\tilde{\xi}_{t}\right\}_{t \geq 0}$ survives when $\bar{N}$ is sufficiently large and this implies the survival of $\left\{\xi_{t}^{i}\right\}_{t \geq 0}$ when $i$ is sufficiently large.

(b) Let $\kappa_{\mathrm{c}}=\kappa_{\mathrm{c}}(\lambda,(1+\phi) / 2)$ be the critical value given in [2, Theorem 5] (see Remark 3.1 for details). Let $\alpha:=\inf _{i \in \mathbb{N}} c_{\infty}(i)$ : by hypothesis, $\alpha>0$ and $\phi \alpha>1$. Choose $\varepsilon>0$ such that $\widetilde{\phi}:=\phi(\alpha-\varepsilon)>1$. By the definition of $c_{\infty}$ and the monotonicity of the sequence of functions $c_{n}$, there exists $n_{\mathrm{c}}$ such that $c_{n}\left(\kappa_{\mathrm{c}}(\lambda, \widetilde{\phi})-1\right)>\alpha-\varepsilon$ for all $n \geq n_{\mathrm{c}}$. Thus, for all $n \geq n_{\mathrm{c}}$, if in a patch there are at most $\kappa_{\mathrm{c}}(\lambda, \widetilde{\phi})$ particles then, for the PRP, the intra-patch reproduction rate is at least $\widetilde{\phi}$. Hence, this PRP dominates the IRP with parameters $\lambda, \widetilde{\phi}$, and $\kappa_{\mathrm{c}}(\lambda, \widetilde{\phi})$, which survives with positive probability.

\section{Open questions}

In the phase diagram the answers to some questions remain unknown. For instance, we do not know if $\lambda_{\mathrm{c}}(\phi, c, N)$ is always equal to 0 when $\phi c(\infty)=1$ (we know that there are examples where this happens). Moreover, we do not know if $\phi_{\mathrm{c}}(\lambda, c, N)>0$ when $\lambda \in\left(1 / 2 d, \lambda_{\mathrm{CP}}\left(\varepsilon_{N}^{d}\right)\right)$. One interesting question is whether a nontrivial invariant measure exists when there is survival with positive probability. The answer is positive if $c(i)=0$ eventually (in this case the configuration space is compact and the invariant measure is obtained as the limit of the process starting from the maximal configuration). In the noncompact case we could try to mimic the technique employed in [6].

On the other hand, we may consider other models which, although similar to those considered here, cannot be obtained as particular cases of the PRP. One of particular interest is a selfregulating IRP where the inter-cluster infection is always active. In each site of $\mathbb{Z}^{d}$ there is a 
(possibly infinite) cluster and, given a regulating function $c$, the transition rates at site $x$ are

$$
\begin{aligned}
& i \rightarrow i+1 \quad \text { at rate }\left(i \phi+\lambda \sum_{z \sim x} \eta(z)\right) c(i) \text { for } 0 \leq i, \\
& i \rightarrow i-1 \quad \text { at rate } i \text { for } i \geq 1 .
\end{aligned}
$$

In the present work, we dealt with the case $c(i)=\max (0,1-i / N)$ (see Subsection 2.3). Further investigations may be made on the process where we add a rate $\delta$ for the transition $i \rightarrow 0$ - the so-called catastrophe or mass extinction.

\section{Acknowledgements}

The authors acknowledge support from the Italian Research Project Prin 2006 - Modelli stocastici a molti gradi di libertà: teoria e applicazioni. The first author is grateful to the Dipartimento di Matematica e Applicazioni, Università di Milano-Bicocca, for their hospitality and logistic support. Moreover, the authors want to thank the anonymous referee for many useful suggestions that helped improve the paper.

\section{References}

[1] Belhadj, L. (2007). Ergodicity and hydrodynamic limits for an epidemic models. Preprint. Available at http://arxiv.org/abs/0710.5185.

[2] Belhadj, L. AND Lanchier, N. (2006). Individual versus cluster recoveries within a spatially structured population. Ann. Appl. Prob. 16, 403-422.

[3] Belhadj, L. And Lanchier, N. (2008). Two-scale contact process and effects of habitat fragmentation on metapopulations. Markov Process. Relat. Fields 14, 487-514.

[4] Bertacchi, D. ANd ZucCa, F. (2009). Approximating critical parameters of branching random walks. J. Appl. Prob. 46, 463-478.

[5] BERTACCHI, D. AND ZuCCA, F. (2009). Characterization of critical values of branching random walks on weighted graphs through infinite-type branching processes. J. Statist. Phys. 134, 53-65.

[6] Bertacchi, D., Posta, G. And Zucca, F. (2007). Ecological equilibrium for restrained random walks. Ann. Appl. Prob. 17, 1117-1137.

[7] Bezuidenhout, C. And Grimmett, G. (1991). Exponential decay for subcritical contact and percolation processes. Ann. Prob. 19, 984-1009.

[8] Bramson, M., Durrett, R. And Swindle, G. (1989). Statistical mechanics of crabgrass. Ann. Prob. 17, 444481.

[9] Durrett, R. And Levin, S. (1994). The importance of being discrete (and spatial). Theoret. Pop. Biol. 46, 363-394.

[10] Harris, T. E. (1974). Contact interactions on a lattice. Ann. Prob. 2, 969-988.

[11] Schinazi, R. B. (2002). On the role of social clusters in the transmission of infectious diseases. Theoret. Pop. Biol. 61, 163-169.

[12] Woess, W. (2000). Random Walks on Infinite Graphs and Groups (Camb. Tracts Math. 138). Cambridge University Press.

[13] Woess, W. (2009). Denumerable Markov Chains. European Mathematical Society, Zürich. 